TRANSACTIONS OF THE

AMERICAN MATHEMATICAL SOCIETY

Volume 175, January 1973

\title{
STEENROD SQUARES IN SPECTRAL SEQUENCES. I
}

\author{
BY
}

WILLIAM M. SINGER

\begin{abstract}
We define two kinds of Steenrod operations on the spectral sequence of a bisimplicial coalgebra. We show the se operations compatible with the differentials of the spectral sequence, and with the Steenrod squares defined on the cohomology of the total complex. We give a general rule for computing the operations on $E_{2}$.
\end{abstract}

The purpose of this paper is to define Stecnrod squaring operations on a class of spectral sequences. In this paper's sequel (hereafter called "II") we describe these actions in some special cases.

Let $\mathcal{O}$ be the category of finite ordered sets and nondecreasing maps. By a bisimplicial coalgebra we mean a purely contravariant functor from $\mathcal{O} \times \mathcal{O}$ to the category of commutative $Z_{2}$ coalgebras. We can regard the vector space dual of any bisimplicial coalgebra as a double cochain complex, and filter it in the usual way. Our main result is that on the $E_{r}$ term of the resulting spectral sequence, for each $r \geq 2$, are defined Steenrod operations

$$
\begin{aligned}
& \mathrm{Sq}^{k}: E_{r}^{p, q} \rightarrow E_{r}^{p, q+k} \quad(0 \leq k \leq q), \\
& \mathrm{Sq}^{k}: E_{r}^{p, q} \rightarrow E_{r}^{p+k-q, 2 q} \quad(k \geq q) .
\end{aligned}
$$

These operations commute with the differentials of the spectral sequence (Proposition 1.4). In particular they are defined on $E_{\infty}$, where they are compatible with the action of Steenrod squares on the cohomology of the total complex (Proposition 1.5).

We thus obtain a larger set of operations than Rector [12] and Smith [15], who consider the related problem of defining Steenrod squares on the spectral sequence of a "mixed" bisimplicial object ..., i.e., a functor from $\mathcal{O}_{\times} \mathcal{O}$ that is contravariant in one variable and covariant in the other. Rector and Smith obtain only operations of type $(0.1)$. Whether operations of type $(0.2)$ can be defined for mixed bisimplicial objects remain an open problem.

Received by the editors September 13, 1971.

AMS (MOS) subject classifications (1970). Primary 55H05, 55G10; Secondary 55H10, $55 \mathrm{H} 20,18 \mathrm{H} 10,18 \mathrm{H} 15,55 \mathrm{~J} 10,55 \mathrm{~F} 35$.

Key words and phrases. Steenrod operation, spectral sequence, cohomology of fiber spaces, cohomology of Hopf algebras, Eilenberg-Moore spectral sequence. 
In II we make a number of applications. The first is to the change of rings spectral sequence, relating the cohomology of an extension of Hopf algebras to the cohomologies of base and fiber. The extensions we consider are in general very bad ... noncentral, algebra twisted, and even coalgebra twisted. Still we are able to define both types of operation $(0.1),(0.2)$ and show how to compute them. Our second application is to the spectral sequence constructed by Moore in [11], passing from the homology of a topological group to the cohomology of its classifying space. Again we compute both kinds of Steenrod squares. Our final application is to the Serre spectral sequence: we use our general constructions to recover the results of [1], [7] and [18].

References are to the bibliography at the end of II. We owe a special debt to Kristensen's work on the Serre sequence [7]. Our Propositions 1.4 and 1.5 are formally identical with the results obtained by Kristensen in the special case.

1. Definitions and results. Our object in this section is to give enough definitions to state precisely the main results of the present paper. We then state the results. Proofs are given in later sections.

We take algebras, coalgebras and Hopf algebras over $Z_{2}$ in the sense of $[10$, Definitions 1.1, 2.1 and 4.1], but we add the convention that all comultiplications are commutative. We write comultiplications $\psi: \Lambda \rightarrow \Lambda \otimes \Lambda$, multiplications $\mu: \Lambda \otimes \Lambda \rightarrow \Lambda$, counits $\epsilon: \Lambda \rightarrow Z_{2}$, units $\eta: Z_{2} \rightarrow \Lambda$. If $\Lambda$ is a Hopf algebra then by a (left) $\Lambda$-coalgebra we mean a coalgebra $M$ that is also a (left) $\Lambda$-module, in such a way that $\psi: M \rightarrow M \otimes M$ and $\epsilon: M \rightarrow Z_{2}$ are $\Lambda$-morphisms. Here $\Lambda$ acts diagonally on $M \otimes M\left[10\right.$, Definition 4.2] and tensor products are always over $Z_{2}$. Similarly we define (left) $\Lambda$-algebras and (left) $\Lambda$-Hopf algebras.

We write $d_{i}^{v}, s_{i}^{v}$ for the "vertical" face and degeneracy operators on a bisimplicial object, and $d_{i}^{b}, s_{i}^{b}$ for the horizontal operators. If $X$ is a bisimplicial object over a category $C$ then by an augmentation for $X$ we mean a simplicial $C$ object $R$, and a morphism of simplicial $C_{\text {objects }} \lambda_{:} X_{0,-} \rightarrow R_{-}$satisfying $\lambda d_{1}^{b}=\lambda d_{0}^{b}: X_{1, q} \rightarrow R_{q}$ for all $q \geq 0$.

Suppose $\Lambda$ a Hopf algebra and $R$ a simplicial $\Lambda$-module. Then we write $C R$ for the $\Lambda$-chain complex defined by $(C R)_{n}=R_{n}, d=\sum_{i=0}^{n} d_{i}$. If $X$ is a bisimplicial $\Lambda$-module we write $C X$ for the double $\Lambda$-chain complex given by $(C X)_{p, q}=$ $X_{p, q}, d^{v}=\Sigma_{i=0}^{q} d_{i}^{v} ; d^{b}=\Sigma_{j=0}^{p} d_{j}^{b}$. We will also regard $C X$ as a $\Lambda$-chain complex with increasing filtration

$$
F_{p}(C X)_{n}=\sum_{i=0}^{p} \bigoplus(C X)_{i, n-i}
$$

Whenever in this paper a double chain complex appears with a single subscript, the subscript refers to total degree.

Suppose $X$ is a bisimplicial $\Lambda$-module and $N$ a $\Lambda$-module. Then we write 
$\operatorname{Hom}_{\Lambda}(C X, N)$ for the $Z_{2}$-cochain complex with decreasing filtration defined by (1.2) $F^{p} \operatorname{Hom}_{\Lambda}^{n}\left(C X, N=\left[f:(C X)_{n}\right) \rightarrow N \mid f\right.$ a $\Lambda$-morphism; $\left.f\left(F_{p-1} C X\right)=0\right]$.

We write $\left(E_{r} \operatorname{Hom}_{\Lambda}(C X, N), d_{r}\right)$, or simply $\left(E_{r}, d_{r}\right)$, for the spectral sequence of this filtered cochain complex, and refer to it as the spectral sequence of the bisimplicial $\Lambda$-coalgebra $X$. If $X$ has an augmentation $\lambda: X \rightarrow R$, then $\lambda$ defines in an obvious way a $Z_{2}$-homomorphism

$$
\lambda^{*}: H^{*}\left(\operatorname{Hom}_{\Lambda}(C R, N)\right) \rightarrow H^{*}\left(\operatorname{Hom}_{\Lambda}(C X, N)\right) .
$$

In the cases of interest to us $\lambda^{*}$ will be an isomorphism. Then $H^{*}\left(\operatorname{Hom}_{\mathbf{\Lambda}}(C R, N)\right)$ is filtered, and is the target of the spectral sequence $\left(E_{r}, d_{r}\right)$. We write

$$
\rho: F^{p} H^{p+q}\left(\operatorname{Hom}_{\Lambda}(C R, N)\right) \rightarrow E_{\infty}^{p, q}
$$

for the usual projection.

We can now state the results. Supposing $\Lambda$ a Hopf algebra, $R$ a simplicial $\Lambda$-coalgebra, $X$ a bisimplicial $\Lambda$-coalgebra, and $N$ a commutative $\Lambda$-algebra. We define products and Steenrod operations in $H^{*}\left(\operatorname{Hom}_{\mathbf{\Lambda}}(C R, N)\right)$, as well as products and Steenrod operations in $H^{*}\left(\operatorname{Hom}_{\Lambda}(C X, N)\right)($ see $(3.8)$ and (3.9)).

Proposition 1.1. Suppose $\lambda: X \rightarrow R$ is an augmentation. Then $\lambda^{*}$ of (1.3) preserves products and Steenrod squares.

Further, we define products in the spectral sequence $\left(E_{r} \operatorname{Hom}_{\Lambda}(C X, N), d_{r}\right)$

$$
E_{r}^{p, q} \otimes E_{r}^{p^{\prime}}, q^{\prime} \rightarrow E_{r}^{p+p^{\prime}, q+q^{\prime}}
$$

(see (4.5)); and Steenrod squares

$$
\begin{aligned}
& \mathrm{Sq}^{k}: E_{r}^{p, q} \rightarrow E_{r}^{p, q+k} \quad(0 \leq k \leq q), \\
& \mathrm{Sq}^{k}: E_{r}^{p, q} \rightarrow E_{r}^{p+k-q, 2 q} \quad(k \geq q) .
\end{aligned}
$$

(See Proposition 4.2.)

Proposition 1.2. Products and Steenrod squares on $E_{2}$ determine products and Steenrod squares on $E_{r}$ for all $r \geq 2$. E.g. if $u \in E_{2}$ survives to $E_{r}$ and represents $[u] \in E_{r}$, then $\mathrm{Sq}^{k} u$ survives to $E_{r}$, and $\left[\mathrm{Sq}^{k} u\right]=\mathrm{Sq}^{k}[u]$.

Proposition 1.3. Under the pairing $(1.5),\left(E_{r}, d_{r}\right)$ is a differential algebra.

Proposition 1.4. Suppose $u \in E_{r}^{p, q}(r \geq 2)$.

Case 1. $k \leq q-r+1$. Then $d_{r} \mathrm{Sq}^{k} u=\overline{\mathrm{Sq}}^{k} d_{r} u$.

Case 2. $q-r+1 \leq k \leq q$. Then $\mathrm{Sq}^{k} u$ survives to a class $\left[\mathrm{Sq}^{k} u\right] \in E_{t}^{p, q+k}$, where $t=2 r+k-q-1$. Also $\mathrm{Sq}^{k} d_{r} u$ survives to a class $\left[\mathrm{Sq}^{k} d_{r} u\right] \in E_{t}^{p+t, 2 q-2 r+2}$, and $d_{t}\left[\mathrm{Sq}^{k} u\right]=\left[\mathrm{Sq}^{k} d_{r} u\right]$. 
Case 3. $q \leq k$. Then $\mathrm{Sq}^{k} u$ survives to a class $\left[\mathrm{Sq}^{k} u\right.$ ] in $E_{t}^{p+k-q, 2 q}$ where $t=2 r-1$. Also $\mathrm{Sq}^{k} d_{r} u$ survives to a class $\left[\mathrm{Sq}^{k} d_{r} u\right.$ ] in $E_{t}^{p+t+k-q, 2 q-2 r+2}$, and $d_{t}\left[\mathrm{Sq}^{k} u\right]=\left[\mathrm{Sq}^{k} d_{r} u\right]$.

Finally we observe that (1.6), (1.7) imply in particular that we have defined squaring operations on $E_{\infty}$. These are related to operations on the target of the spectral sequence:

Proposition 1.5. Suppose $X$ a bisimplicial $\Lambda$-coalgebra with augmentation $\lambda: X \rightarrow R$, suppose $N$ a commutative $\Lambda$-algebra, and suppose $\lambda^{*}$ of (1.3) an isomorphism. Suppose $u \in F^{p} H^{p+q}\left(\operatorname{Hom}_{\Lambda}(C R, N)\right)$ and $v \in F^{r} H^{r+s}\left(\operatorname{Hom}_{\Lambda}(C R, N)\right)$. Then $u \cdot v \in F^{p+r} H^{*}\left(\operatorname{Hom}_{\Lambda}(C R, N)\right)$, and $\rho(u \cdot v)=\rho u \cdot \rho v(\rho$ as in (1.4)). Further:

Case 1. $0 \leq k \leq q$. Then $\mathrm{Sq}^{k} u \in F^{p} H^{*}\left(\operatorname{Hom}_{\Lambda}(C R, N)\right)$ and $\rho \mathrm{Sq}^{k} u=\mathrm{Sq}^{k} \rho u$.

Case 2. $q \leq k$. Then $\mathrm{Sq}^{k} u \in F^{p+k-q} H^{*}\left(\operatorname{Hom}_{\Lambda}(C R, N)\right)$ and $\rho \mathrm{Sq}^{k} u=\mathrm{Sq}^{k} \rho u$.

The rest of this paper is devoted to proving these results, as well as some auxiliary ones. (See in particular Proposition 5.1, which gives a useful description of the products and Steenrod squares on $E_{2}$.)

2. Simplicial Eilenberg-Zilber maps. Suppose for each integer $k \geq 0$ and for every pair of simplicial $Z_{2}$-modules $R, S$ we have defined a $Z_{2}$-homomorphism $D_{k}=D_{k}(R, S): C(R \times S) \rightarrow C R \otimes C S$ homogeneous of degree $k$ and natural in the variables $R$ and $S$; suppose that for all $k \geq 0$

$$
d D_{k}+D_{k} d=D_{k-1}+T D_{k-1} T
$$

where $T: C(R \times S) \rightarrow C(S \times R) ; T: C R \otimes C S \rightarrow C S \otimes C R$ are the switching maps (read $\left.D_{-1}=0\right)$. Suppose finally that $D_{0}: C(R \times S)_{0} \rightarrow(C R \otimes C S)_{0}$ is the identity. Then we call the sequence $\left(D_{k}\right)$ a simplicial Eilenberg-Zilber map. The existence of such a sequence is proved by Dold [3].

Suppose now $\Lambda$ is a Hopf algebra. If $R, S$ are simplicial $\Lambda$-modules we write $R \times S$ for the simplicial $\Lambda$-module $(R \times S)_{p}=R_{p} \otimes S_{p}$ with diagonal $\Lambda$-action. The tensor product $C R \otimes C S$ of $\Lambda$-chain complexes is again a $\Lambda$-chain complex, with diagonal $\Lambda$-action.

Proposition 2.1. Suppose $\left(D_{k}\right)$ a simplicial Eilenberg-Zilber map. Suppose $\Lambda$ a Hopf algebra and $R$ and $S$ simplicial $\Lambda$-modules. Then $D_{k}: C(R \times S) \rightarrow$ $C R \otimes C S$ is a $\Lambda$-bomomorphism.

This follows easily from the functorial properties of $D_{k}$. As a consequence we obtain for any $\Lambda$-module $N$ and any simplicial $\Lambda$-modules $R, S$ the $Z_{2}$-homomorphisms $D_{k}^{*}: \operatorname{Hom}_{\Lambda}(C R \otimes C S, N) \rightarrow \operatorname{Hom}_{\Lambda}(C(R \times S), N)$ satisfying the dual of (2.1).

Now suppose $N$ a commutative $\Lambda$-algebra, and $R$ a simplicial $\Lambda$-coalgebra. We define a map of cochain complexes 


$$
\phi: \operatorname{Hom}_{\Lambda}(C R, N) \otimes \operatorname{Hom}_{\Lambda}(C R, N) \rightarrow \operatorname{Hom}_{\Lambda}(C R \otimes C R, N)
$$

by assigning to the pair $x: C R \rightarrow N, y: C R \rightarrow N$, the cochain $C R \otimes C R \stackrel{x \otimes y}{\longrightarrow}$ $N \otimes N \stackrel{\mu}{\longrightarrow} N$. We then define cochain operations $\mu: \operatorname{Hom}_{\Lambda}^{p}(C R, N) \otimes \operatorname{Hom}_{\Lambda}^{q}(C R, N)$ $\rightarrow \operatorname{Hom}_{\Lambda}^{p+q}(C R, N)$ and $S^{k}: \operatorname{Hom}_{\Lambda}^{n}(C R, N) \rightarrow \operatorname{Hom}_{\Lambda}^{n+k}(C R, N)$ by the customary formulas $\mu(x \otimes y)=\psi^{*} D_{0}^{*} \phi(x \otimes y) ; S^{k} x=\psi^{*} D_{n-k}^{*} \phi(x \otimes x)+\psi^{*} D_{n-k+1}^{*} \phi(x \otimes \delta x)$. Here $\psi: R \rightarrow R \times R$ is the coproduct. Then $\mu$ and $S^{k}$ define products and Steenrod squares on $H^{*}\left(\operatorname{Hom}_{\Lambda}(C R, N)\right)$. These satisfy all the usual rules, although both Cartan formula and Adem relations must be interpreted with $\mathrm{Sq}^{0} \neq 1$.

The notion of a "special" Eilenberg-Zilber map will be useful to us in $\$ 4$.

Definition 2.2. A simplicial Eilenberg-Zilber map $\left(D_{k}\right)$ is called "special" if for all pairs of simplicial $Z_{2}$-modules $R, S$; and all integers $n, k$ :

(1) The image of $D_{k}: C(R \times S)_{n} \rightarrow(C R \otimes C S)_{n+k}$ is contained in the subspace $\Sigma_{i, j \leq n} \bigoplus\left((C R)_{i} \otimes(C S)_{j}\right)$. In particular, $D_{k}\left(C(R \times S)_{n}\right)=0$ if $k>n$.

(2) The map $D_{n}: C(R \times S)_{n} \rightarrow(C R \otimes C S)_{2 n}$ is given by $D_{n}(\sigma \otimes r)=\sigma \otimes r$ for all $\sigma \in R_{n}, \tau \in S_{n}$.

\section{Proposition 2.3. There exists a special Eilenberg-Zilber map.}

The proof is an easy modification of Spanier [16, p. 274].

3. Bisimplicial Eilenberg-Zilber maps. Suppose $\Lambda$ a Hopf algebra and $X, Y$ bisimplicial $\Lambda$-modules. We define a double $\Lambda$-complex called the "vertical tensor product": $\left(C X \otimes_{V} C Y\right)_{p, q}=\Sigma_{k+l=q} \bigoplus\left(X_{p, k} \otimes Y_{p, l}\right)$. Here the horizontal differential is $\Sigma_{i} d_{i}^{b} \otimes d_{i}^{b}$, and the vertical differential is $d^{v} \otimes \mathrm{id}+\mathrm{id} \otimes d^{v}$. We define the ordinary tensor product of the double complexes $C X, C Y$ by writing $(C X \otimes C Y)_{p, q}=\Sigma_{i+j=p} \Sigma_{k+l=q} \bigoplus\left(X_{i, k} \otimes Y_{j, l}\right)$ (action of $\Lambda$ is always diagonal).

Fix a simplicial Eilenberg-Zilber map $\left(D_{k}\right)$. Define $\Lambda$-homomorphisms:

$$
\begin{aligned}
& G_{s}: C(X \times Y) \rightarrow C X \otimes_{V} C Y, \\
& H_{r}: C X \otimes_{V} C Y \rightarrow C X \otimes C Y,
\end{aligned}
$$

where $G_{s}$ is homogeneous of bidegree $(0, s)$ and $H_{r}$ is homogeneous of bidegree $(r, 0)$, as follows. The restriction of $G_{s}$ to $C(X \times Y)_{p, q}$ is

$$
D_{s}\left(X_{p,-} ; Y_{p,-}\right): X_{p, q} \otimes Y_{p, q} \rightarrow \sum_{k+l=q+s} \oplus\left(X_{p, k} \otimes Y_{p, l}\right)
$$

and the restriction of $H_{r}$ to the summand $X_{p, k} \otimes Y_{p, l}$ of $\left(C X \otimes_{V} C Y\right)_{p, q}$ is $(k+l=q)$ :

Clearly

$$
D_{r}\left(X_{-, k} ; Y_{-, l}\right): X_{p, k} \otimes Y_{p, l} \rightarrow \sum_{i+j=p+r} \oplus\left(X_{i, k} \otimes Y_{j, l}\right) .
$$




$$
\begin{aligned}
& d^{v} G_{s}+G_{s} d^{v}=G_{s-1}+T G_{s-1} T, \\
& d^{b} H_{r}+H_{r} d^{b}=H_{r-1}+T H_{r-1} T, \\
& d^{b} G_{s}=G_{s} d^{b} ; \quad d^{v} H_{r}=H_{r} d^{v} .
\end{aligned}
$$

We now define for each integer $k \geq 0$ a $\Lambda$-homomorphism $K_{k}: C(X \times Y) \rightarrow$ $C X \otimes C Y$ homogeneous of degree $k$ with respect to total degree by setting

$$
K_{k}=\sum_{r=k} T^{s} H_{r} T^{s} G_{s}
$$

where $T^{s}$ is the $s$-fold iterate of the switching morphism. From the relations (3.3) we find after a short computation

$$
d K_{k}+K_{k} d=K_{k-1}+T K_{k-1} T
$$

where the $d$ 's are total differentials. Thus $\left(K_{k}\right)$ can be called a bisimplicial Eilenberg-Zilber map.

It is now apparent how to define cohomology operations on $H^{*}\left(\operatorname{Hom}_{\mathbf{\Lambda}}(C X, N)\right)$ for any bisimplicial $\Lambda$-coalgebra $X$ and any commutative $\Lambda$-algebra $N$. Each $K_{k}$ defines

$$
K_{k}^{*}: \operatorname{Hom}_{\Lambda}(C X \otimes C Y, N) \rightarrow \operatorname{Hom}_{\Lambda}(C(X \times Y), N)
$$

and we set up a map of cochain complexes

$$
\phi: \operatorname{Hom}_{\Lambda}(C X, N) \otimes \operatorname{Hom}_{\Lambda}(C X, N) \rightarrow \operatorname{Hom}_{\Lambda}(C X \otimes C X, N)
$$

by analogy with (2.2). We define cochain operations $\mu$ : $\operatorname{Hom}_{\Lambda}^{p}(C X, N) \otimes$ $\operatorname{Hom}_{\Lambda}^{q}(C X, N) \rightarrow \operatorname{Hom}_{\Lambda}^{p+q}(C X, N), S^{k}: \operatorname{Hom}_{\Lambda}^{n}(C X, N) \rightarrow \operatorname{Hom}_{\Lambda}^{n+k}(C X, N)$ by

$$
\begin{gathered}
\mu(x \otimes y)=\psi^{*} K_{0}^{*} \phi(x \otimes y), \\
S^{k} x=\psi^{*} K_{n-k}^{*} \phi(x \otimes x)+\psi^{*} K_{n-k+1}^{*} \phi(x \otimes \delta x) .
\end{gathered}
$$

Then $\mu$ and $S^{k}$ pass to products and Steenrod squares on $H^{*}\left(\operatorname{Hom}_{\mathbf{\Lambda}}(C X, N)\right)$.

These operations are independent of the choice of $\left(D_{k}\right)$, and satisfy Cartan formulas and Adem relations. We do not prove these statements here, as we do not need them. The reader can construct his own proofs, by using an analogue of the Eilenberg-Zilber theorem which compares $C(X \times Y)$ to $C X \otimes C Y$, where $X, Y$ are bisimplicial objects.

Proof of Proposition 1.1. Suppose $X$ a bisimplicial $\Lambda$-coalgebra, $R$ a simplicial $\Lambda$-coalgebra, and $\lambda: X \rightarrow R$ an augmentation. Suppose $x: R_{n} \rightarrow N$ is a cocycle representing $u \in H^{n}\left(\operatorname{Hom}_{\Lambda}(C R, N)\right)$. Then $\mathrm{Sq}^{k} \lambda^{*} u$ is represented by $\psi^{*} K_{n-k}^{*} \phi\left(\lambda^{*} x \otimes \lambda^{*} x\right):(C X)_{n+k} \rightarrow N$. Using (3.1), (3.2), (3.4) and the fact that 
$H_{0}=D_{0}\left(X_{-, n} ; X_{-, n}\right): X_{0, n} \otimes X_{0, n} \rightarrow X_{0, n} \otimes X_{0, n}$ is the identity, we find that $\mathrm{Sq}^{k} \lambda^{*} u$ is represented by

$$
\begin{gathered}
X_{0, n+k} \stackrel{\Psi}{\rightarrow} X_{0, n+k} \otimes X_{0, n+k} \stackrel{D_{n-k}^{n, n}\left(X_{0,-} ; X_{0,-}\right)}{\longrightarrow} X_{0, n} \otimes X_{0, n} \\
\stackrel{\lambda \otimes \lambda}{\longrightarrow} R_{n} \otimes R_{n} \stackrel{x \otimes x}{\longrightarrow} N \otimes N \stackrel{\mu}{\longrightarrow} N
\end{gathered}
$$

where we have written $D_{n-k}^{n, n}$ for the map $D_{n-k}$ followed by projection to the factor $X_{0, n} \otimes X_{0, n} \subset\left(C X \otimes_{V} C X\right)_{0,2 n^{*}}$ But $D_{n-k}^{n, n}$ is natural with respect to the map of simplicial objects $\lambda: X_{0,-} \rightarrow R_{-}$, and since $\lambda$ preserves the coproduct we can write (3.10) in the form $\mu(x \otimes x) D_{n-k}^{n, n}(R, R) \psi \lambda: X_{0, n+k} \rightarrow N$. But this cochain represents $\lambda^{*} \mathrm{Sq}^{k} u$. A similar argument shows $\lambda^{*}$ preserves products.

4. The spectral sequence of a bisimplicial $\Lambda$-coalgebra. If $X, Y$ are bisimplicial $\Lambda$-coalgebras, we give to $C X \otimes C Y$ the usual (increasing) filtration associated with a tensor product of filtered complexes. Then $\operatorname{Hom}_{\Lambda}(C X \otimes C Y, N)$ has the dual, decreasing filtration (as in (1.2)). Then $\phi$ of (3.7) is filtration preserving. Further

Proposition 4.1. Suppose the simplicial Eilenberg-Zilber map $\left(D_{k}\right)$ is special. Then $K_{k} F_{p} C(X \times Y) \subset F_{p+k}(C X \otimes C Y)$, and $K_{k} F_{p} C(X \times Y) \subset F_{2 p}(C X \otimes C Y)$.

Proof. The first statement follows from the definition (3.4) of $K_{k}$, and the second follows from (1) of Definition 2.2.

Consequently the $Z_{2}$-homomorphisms $K_{k}^{*}$ of (3.6) satisfy

$$
\begin{gathered}
K_{k}^{*} F^{p} \subset F^{1 \mathrm{lig}(p / 2)}, \\
K_{k}^{*} F^{p} \subset F^{p-k}
\end{gathered}
$$

where lig $(x)$ denotes the least integer greater than or equal to $x$. From now on we assume that $\left(D_{k}\right)$ is special.

The spectral sequence $E_{r}=E_{r} \operatorname{Hom}_{\Lambda}(C X, N)(N$ a commutative $\Lambda$-algebra $)$ is obtained by the usual formulas:

$$
E_{r}^{p, q}=Z_{r}^{p, q} /\left[\delta Z_{r-1}^{p-r+1, q+r-2}+Z_{r-1}^{p+1, q-1}\right]
$$

where

$$
Z_{r}^{p, q}=\left[x \in F^{p} \operatorname{Hom}_{\Lambda}^{p+q}(C X, N) \mid \delta x \in F^{p+r} \operatorname{Hom}_{\Lambda}(C X, N)\right] .
$$

We write $Z_{r}^{p}=\Sigma_{q} \bigoplus Z_{r}^{p, q} ; E_{r}^{p}=\Sigma_{q} \oplus E_{r}^{p, q}$. The differential $\delta$ on $\operatorname{Hom}_{\boldsymbol{\Lambda}}(C X, N)$ induces $d_{r}: E_{r}^{p, q} \rightarrow E_{r}^{p+r, q-r+1}$.

We define the product $(1.5)$ by the composition 


$$
\begin{aligned}
E_{r} \operatorname{Hom}_{\Lambda}(C X, N) \otimes E_{r} \operatorname{Hom}_{\Lambda}(C X, N) & \rightarrow E_{r}\left(\operatorname{Hom}_{\Lambda}(C X, N) \otimes \operatorname{Hom}_{\Lambda}(C X, N)\right) \\
& \stackrel{\mu_{*}}{\rightarrow} E_{r}\left(\operatorname{Hom}_{\Lambda}(C X, N)\right)
\end{aligned}
$$

where $\mu_{*}$ is the map of spectral sequences induced by the map $\mu: \operatorname{Hom}_{\Lambda}(C X, N)$ $\otimes \operatorname{Hom}_{\Lambda}(C X, N) \rightarrow \operatorname{Hom}_{\Lambda}(C X, N)$ of filtered complexes given by (3.8). Steenrod squares in the spectral sequence are defined by virtue of

Proposition 4.2. Suppose $r>2$ and $x \in Z^{p, q}$.

Case 1. $0 \leq k \leq q$. Then $S^{k} x \in Z_{r}^{p, q+k}\left(S^{k}\right.$ as in (3.9)) and the correspondence $x \rightarrow S^{k} x$ passes to a homomorphism $\mathrm{Sq}^{k}: E_{r}^{p, q} \rightarrow E_{r}^{p, q+k}$.

Case 2. $q \leq k$. Then $S^{k} x \in Z^{p+k-q, 2 q}$, and the correspondence $x \rightarrow S^{k} x$ passes to a bomomorpbism $\mathrm{Sq}^{k}: E_{r}^{p, q} \rightarrow E_{r}^{p+k-q, 2 q}$.

Proof. The proofs of these statements depend only on the definition (3.9) and the properties (3.5), (4.1), (4.2) of $K_{k}$. Case 1 requires (4.1) and Case 2 requires (4.2). We write out the proof for Case 2. If $x \in Z_{r}^{p, q}$ then $\phi(x \otimes x) \epsilon$ $F^{2 p} \operatorname{Hom}_{\Lambda}(C X \otimes C X, N)$, so from (4.2) we have $\psi^{*} K_{n-k}^{*} \phi(x \otimes x) \epsilon$ $F^{p-q+k} \operatorname{Hom}_{\Lambda}(C X, N)$; here we have set $n=p+q$. Also $\delta x \epsilon$ $F^{p+r} \operatorname{Hom}_{\Lambda}(C X, N)$ so

$$
\psi^{*} K_{n-k+1}^{*} \phi(x \otimes \delta x) \in F^{p-q+k+r-1} \operatorname{Hom}_{\Lambda}(C X, N) \subset F^{p-q+k} \operatorname{Hom}_{\Lambda}(C X, N) \quad(r \geq 1) 。
$$

Hence $S^{k} x \in F^{p-q+k} \operatorname{Hom}_{\Lambda}(C X, N)$. Further $\delta S^{k} x=S^{k} \delta x=\psi^{*} K_{n-k+1}^{*} \phi(\delta x \otimes \delta x)$ which by (4.2) lies in $F^{p+k-q+r} \operatorname{Hom}_{\Lambda}(C X, N)$. This proves $S^{k} x \in Z_{r}^{p+k-q}$.

We claim next that if $x, y \in Z_{r}^{p, q}$ then $S^{k}(x+y)-S^{k} x-S^{k} y$ represents zero in $E_{r}^{p+k-q}$. In fact (3.5) and (3.9) imply

(4.6) $S^{k}(x+y)-S^{k} x-S^{k} y=\psi^{*}\left[\delta K_{n-k+1}^{*} \phi(x \otimes y)+K_{n-k+1}^{*} \phi(1+T)(\delta x\right.$ ஓ $\left.y)\right]$.

But use of (4.2) shows that the first term on the right of (4.6) lies in $\delta Z_{r-1}^{p+k-q-r+1}$ and that the second term lies in $Z_{r-1}^{p+k-q+1}$. Hence both terms represent zero in $E_{r}^{p+k-q}$

We claim finally that $S^{k}$ passes to a well-defined map $E_{r}^{p, k} \rightarrow E_{r}^{p+k-q, 2 q}$. In view of our analysis of (4.6) we need only show that if $x \in Z_{r-1}^{p+1, q-1}$ then $S^{k} x \in Z_{r-1}^{p+k-q+1}$, and that if $x \in \delta Z_{r-1}^{p-r+1, q+r-2}$ then $S^{k} x \in \delta Z_{r-1}^{p+k-q-r+1}$. But both these statements follow easily from (4.2) and the relation $\delta S^{k}=S^{k} \delta$.

Proof of Proposition 1.2. Immediate from the definitions.

Proof of Proposition 1.3. Immediate.

Proof of Proposition 1.4. All the statements are consequences of the relation $\delta S^{k}=S^{k} \delta$. We write out the proof for Case 2. Suppose $x \in Z_{r}^{p, q}$ represents $u \in E_{r}^{p, q}$. Then $\delta x \in F^{p+r} \operatorname{Hom}_{\Lambda}(C X, N)$, and by repeating an argument in the proof of Proposition 4.2 we find $S^{k} \delta x \in F^{p+t} \operatorname{Hom}_{\Lambda}(C X, N)$, where $t=2 r-q+k-1$. 
Since $\delta S^{k} x=S^{k} \delta x$ we find $S^{k} x \in Z_{t}^{p}$. Hence $\mathrm{Sq}^{k} u$ survives to $E_{t}$. Next we observe that $\mathrm{Sq}^{k} d_{r} u$ is represented in $Z_{r}^{p+t}$ by $S^{k} \delta x$. But this element surely lies in $Z_{t}^{p+t}$, since $\delta S^{k} \delta x=S^{k} \delta \delta x=0$. Hence $\mathrm{Sq}^{k} d_{r} u$ survives to $E_{t}$. Finally, the relation $\delta S^{k}=S^{k} \delta$ implies $d_{t}\left[\mathrm{Sq}^{k} u\right]=\left[\mathrm{Sq}^{k} d_{r} u\right]$.

Proof of Proposition 1.5. Let $\underline{\rho}: F^{p} H^{p+q}\left(\operatorname{Hom}_{\Lambda}(C X, N)\right) \rightarrow E_{\infty}^{p, q}$ be the standard projection. Then $\rho=\rho \lambda^{*}$. To show that $\rho$ commutes with products and Steenrod squares it suffices, in view of Proposition 1.1, to show that $\underline{\rho}$ does. But this is immediate from the definitions (Proposition 4.2).

5. Operations on $E_{2}$. One representation of $E_{2}$ is given by (4.3), but it is sometimes more convenient to work with

$$
E_{2}^{p, q}=H_{b}^{p} H_{v}^{q}
$$

Here the right-hand side is the pth homology group of the complex

$$
\ldots \rightarrow H^{q}\left(\operatorname{Hom}_{\Lambda}\left((C X)_{p,-} ; N\right)\right) \stackrel{\delta^{b}}{\rightarrow} H^{q}\left(\operatorname{Hom}_{\Lambda}\left((C X)_{p+1,-} ; N\right)\right) \rightarrow \cdots
$$

Using (5.1) we can represent and element of $E_{2}$ by a $\Lambda$-homomorphism $x: X_{p, q}$ $\rightarrow N$ satisfying $\delta^{v} x=0$ and $\delta^{b} x=\delta^{v} y$ for some $y: X_{p+1, q-1} \rightarrow N$. The next result tells how to compute Steenrod squares on $E_{2}$, using this representation

Notation. If $\left(D_{k}\right)$ is a (special) Eilenberg-Zilber map, and if $R, S$ are simplicial $\Lambda$-modules, then for any pair of integers $(i, j)$ for which $i+j=p+k$ write $D_{k}^{i, j}(R, S): R_{p} \otimes S_{p} \rightarrow R_{i} \otimes S_{j}$ for the composition

$$
R_{p} \otimes S_{p} \stackrel{D_{k}(R, S)}{\longrightarrow}(C R \otimes C S)_{p+k} \stackrel{\text { project }}{\longrightarrow} R_{i} \otimes S_{j}
$$

Proposition 5.1. Suppose $x: X_{p, q} \rightarrow N, y: X_{r, s} \rightarrow N$ are $\Lambda$-bomomorphisms representing $u \in E_{2}^{p, q}, v \in E_{2}^{r, s}$. Then $u \cdot v \in E_{2}^{p+r, q+s}$ is represented by the composition

$$
X_{p+r, q+s} \stackrel{\psi}{\rightarrow} X_{p+r, q+s} \otimes X_{p+r, q+s} \stackrel{D_{0}^{q, s}\left(X_{p+r,-} ; X_{p+r,-}\right)}{\longrightarrow} X_{p+r, q} \otimes X_{p+r, s}
$$

$$
\stackrel{D_{0}^{p, r}\left(X_{-, q} ; X_{-, s}{ }^{\prime}\right.}{\longrightarrow} X_{p, q} \otimes X_{r, s} \stackrel{x \otimes y}{\longrightarrow} N \otimes N \stackrel{\mu}{\rightarrow} N
$$

If $k \leq q$ then $\mathrm{Sq}^{k} u \in E_{2}^{p, q+k}$ is represented by the composition

$$
\begin{aligned}
X_{p, q+k} \stackrel{\psi}{\rightarrow} X_{p, q+k} \otimes X_{p, q+k} & \\
& \stackrel{D_{q-k}^{q, q}\left(X_{p,-} ; X_{p,-}\right)}{\longrightarrow} X_{p, q} \otimes X_{p, q} \stackrel{x \otimes x}{\longrightarrow} N \otimes N \stackrel{\mu}{\rightarrow} N,
\end{aligned}
$$

and if $k \geq q, \mathrm{Sq}^{k} u \in E_{2}^{p+k-q, 2 q}$ is represented by the composition 


$$
\begin{aligned}
X_{p+k-q, 2 q} \stackrel{\uplus}{\rightarrow} X_{p+k-q, 2 q} \otimes X_{p+k-q, 2 q} \\
\\
\stackrel{D_{0}^{q, q}\left(X_{p+k-q,-} ; X_{p+k-q,-}\right)}{\longrightarrow} X_{p+k-q, q} \otimes X_{p+k-q, q .} \\
\stackrel{D_{p+q-k}^{p, p}\left(X_{-, q} ; X_{-, q}\right)}{\longrightarrow} X_{p, q} \otimes X_{p, q} \stackrel{x \otimes x}{\longrightarrow} N \otimes N \stackrel{\mu}{\rightarrow} N .
\end{aligned}
$$

Proof. We give the proof for (5.2); the others are similar. The Steenrod squares on $E_{r}$ were originally defined in terms of the representation (4.3). In that language $u$ is represented by a cochain of the form $x+y$, for some $y \in$ $F^{p+1} \operatorname{Hom}_{\Lambda}(C X, N)$, and $\mathrm{Sq}^{k} u$ is represented by $S^{k}(x+y) \in \Sigma_{i 2 p} \bigoplus \operatorname{Hom}_{\Lambda}\left(X_{i, j} ; N\right)$. To translate back to the language of (5.1) we need only pick out from $S^{k}(x+y)$ the component that lies in $\operatorname{Hom}_{\Lambda}\left(X_{p, q+k} ; N\right)$. But use of (4.1) shows that terms in $S^{k}(x+y)$ involving either $y$ or $\delta x$ (such as $K_{n-k}^{*} \phi(x \otimes y)$ or $K_{n-k+1}^{*} \phi(x \otimes \delta x)$ ) make no contribution to the crucial component. Thus it suffices to find the appropriate component of $\psi^{*} K_{n-k}^{*} \phi(x \otimes x)(n=p+q)$. Use of (3.1), (3.2), (3.4) shows that this is the composition

$$
\mu(x \otimes x) D_{p}^{p, p}\left(X_{-, q} ; X_{-, q}\right) D_{q-k}^{q, q}\left(X_{p,-} ; X_{p,-}\right) \psi: X_{p, q+k} \rightarrow N .
$$

But by virtue of (2) of Definition 2.2, $D_{p}^{p, p}\left(X_{-, q} ; X_{-, q}\right)$ is the identity map on $X_{p, q} \otimes X_{p, q^{\bullet}}$ So (5.4) reduces to (5.2).

Proposition 5.1 can be used to show that the Steenrod squares on $E_{2}$ (and hence on $E_{r}$ for all $r \geq 2$ ) are independent of the choice of special EilenbergZilber map. It can. also be used to prove Adem relations and Cartan formulas. But in II we will obtain these results with no extra work, for each of the special cases we consider. For this reason we omit the general proofs. 